\section{Turning intestines into insulin factories}

The transcription factor Foxol mediates responses to hormones and nutrients in addition to regulating terminal differentiation of various tissues. Particularly intriguing is the finding that ablating Foxol leads to an increased number of beta cell precursors (Neurog $3^{+}$cells) in cultured pancreatic epithelium. Now Talchai et al. show that ablating Foxol in mice (NKO mice) generates beta cell-producing Neurog $3^{+}$cells in the adult gut as well. This was a somewhat surprising result; although intestinal Neurog $3^{+}$cells and pancreatic Neurog $3^{+}$cells have a common endodermal origin, the gut precursor cells normally give rise only to enterendocrine precursors. However, in NKO mice, insulin, along with several beta cell markers, was detected in both neonatal and adult intestines. Furthermore, the insulin-secreting cells from the intestines were glucose responsive both in vitro and in vivo in a toxin-induced diabetes mouse model. One hundred percent of toxin-treated wild-type mice required insulin to survive, whereas $75 \%$ of NKO animals survived after insulin withdrawal. These findings suggest that pharmacological interventions that knock down Foxol levels could someday replace insulin administration in type 1 diabetics. (Nat. Genet. doi: 10.1038/ng.2215, advance online publication, 11 March 2012)

\section{How sulfa drugs work}

The catalytic and resistance mechanisms of bacterial dihydropteroate synthase (DHPS), the target of the sulfa drug antibiotics, have finally been solved thanks to work by Yun et al. Sulfonamides were among the first antibiotics discovered and are potent against bacteria and some eukaryotic pathogens, but resistance arising through mutations in DHPS has severely limited use of these drugs in recent years. DHPS is a key enzyme in the folic acid biosynthesis pathway, and it catalyzes the production of the folate intermediate 7,8-dihydropteroate from 6-hydroxymethyl-7,8-dihydropterin pyrophosphate and $p$-aminobenzoic acid (PABA). Sulfa drugs act by mimicking PABA and binding to DHPS. First, the authors used computational predictions of enzyme reactions and crystallization of DHPS enzymes from Bacillus anthracis and Yersinia pestis to establish an essential role for bound magnesium in catalysis. Then, using structural enzymology techniques that take advantage of the fact that many proteins retain catalytic activity in a crystalline form, and by soaking DHPS crystals from Y. pestis in substrates and substrate analogs, the authors deduced the complete catalytic mechanism, including where DHPS contacts sulfa drugs. This should pave the way for rational design of new sulfa drugs. (Science 335, 1110-1114, 2012)

SJ

\section{Reverse plant breeding success}

Creation of F1 hybrid varieties that typically produce better yields is traditionally achieved by breeding homozygous parent plants. Aside from the time breeding takes, uncharacterized heterozygotes cannot easily be

Written by Kathy Aschheim, Laura DeFrancesco, Susan Jones \& Jason Kreisberg converted into homozygous parental breeding lines, as desirable allele combinations are lost through recombination during meiosis. That obstacle has been overcome by Wijnker et al. who have reverse bred Arabidopsis thaliana parental lines from heterozygous hybrids using a process that involves preventing meiotic crossing over. The authors used RNA interference (RNAi) to silence DISRUPTED MEIOTIC cDNA1 (DMC1) to reduce the genetic variation in the spores. The pollen was used to generate reverse-breeding haploids from which fertile double haploids were grown (double the number of chromosomes). By crossing complementary pairs of double haploids, they recreated the original heterozygous hybrid. A key advantage of this method is the possibility of obtaining breeding lines for any heterozygote. Crops in which this technology might prove useful include cucumber, sorghum and rice, but it won't be suitable for plants with more than 12 chromosomes, such as potato and soybean. (Nat. Genet. doi:10.1038/ng.2203, advance online publication, 11 March 2012)

\section{Starving HIV of genome building blocks}

SAMHD1 is the latest in the ever-growing list of HIV restriction factors. A new study by Lahouassa et al. shows that this host factor prevents HIV from infecting dendritic and other myeloid cells by reducing levels of intracellular dNTPs. When SAMHD1 was first identified as an HIV restriction factor in 2011, its mechanism of action was unclear. Subsequently, it was identified as a deoxynucleoside triphosphate triphosphohydrolase, which converts dNTPs into deoxynucleosides and inorganic triphosphates. Lahouassa et al. now link this enzymatic activity to suppression of HIV. Knocking down SAMHD1 in myeloid cells increased free dNTP levels, rendering the cells permissive for HIV infection. Conversely, increased expression of SAMHD1 decreased both dNTP levels and permissiveness to HIV infection. This work reveals a new facet of innate immunity: viruses that replicate through a DNA intermediate can be inhibited by limiting levels of free dNTPs. Thus, drugs that decrease available dNTP pools may be therapeutically useful not only for retroviruses but also for adenoviruses, papillomaviruses and herpesviruses. (Nat. Immunol. 13, 223-228, 2012)

$J K$

\section{Nanodevices measure bioelectricity}

Cells have been grown on arrays of microscale electrodes, which can both inject and record cellular currents, to study their electrical activity. Three recent papers have measured cellular currents using much smaller, nanoscale electrodes. Robinson et al. fabricated electrodes made of doped, conducting silicon $\sim 150 \mathrm{~nm}$ in diameter and $3 \mu \mathrm{m}$ high, whereas Xie et al. worked with platinum electrodes $\sim 150 \mathrm{~nm}$ in diameter and 1-2 $\mu \mathrm{m}$ high. In both studies, the amplitude of the action potentials recorded from neurons or cardiomyocytes was only $\sim 10 \%$ of that measured by patch clamp, the gold-standard electrophysiology technique, although unlike patch clamp, the nanoscale electrode arrays allow recording of many cells over several days. In contrast, Duan et al. achieved much higher sensitivity by using a silicon fieldeffect transistor (FET) with a branched nanotube, measuring $\sim 55$ $\mathrm{nm}$ at the tip and $1-1.5 \mu \mathrm{m}$ in height. With this approach, the amplitude of action potentials was similar to that seen with patch clamp. This FET-based active recording requires only a single nanotube, in contrast to the multiple, larger nanowires contained in each passive electrode in the other two studies. Compared with microscale electrodes, nanoscale electrodes are less damaging to cells and could be arrayed in closer proximity to increase the resolution of the technique. (Nat. Nanotechnol. 7, 180-184, 2012; Nat. Nanotechnol. 7, 185-190, 2012; Nat. Nanotechnol. 7, 174-179, 2012)

KA 